\title{
Effects of Roystonea Regia (D-004) and Saw Palmetto Lipid Extracts in Men with Symptomatic benign Prostatic Hyperplasia
}

\author{
Raúl Guzmán, ${ }^{1}$ Ramiro Fragas, ${ }^{2}$ José Illnait, ${ }^{3}$ Rosa Mas, ${ }^{3}$ Lilia Fernández, ${ }^{3}$ \\ Manuel Pedroso, ${ }^{1}$ Jilma Mena, ${ }^{1}$ Ana T. Rodríguez, ${ }^{1}$ Sarahí Mendoza, ${ }^{3}$ Esther \\ Enrique, ${ }^{1}$ Raiza Martinez, ${ }^{1}$ Julio Fernández, ${ }^{3}$ Rafael Gámez, ${ }^{3}$ \\ Lisete Borrero, ${ }^{4}$ Dalmer Ruiz. ${ }^{5}$ \\ ${ }^{I}$ Dr. Salvador Allende Hospital, Havana City, Cuba. \\ ${ }^{2}$ Dr. Manuel Fajardo Hospital, Havana City, Cuba. \\ ${ }^{3}$ Centre of Natural Products, National Centre for Scientific Research, Havana City, Cuba. \\ ${ }^{4}$ Medical Surgical Research Centre, Havana City, Cuba. \\ ${ }^{5}$ Software and Database Group from the National Centre for Scientific Research, Havana City, Cuba
}

\begin{abstract}
:
Background: Benign prostatic hyperplasia (BPH), common among elderly men, often causes lower urinary tract symptoms (LUTS). BPH/LUTS pharmacological therapies include $5 \alpha$-reductase inhibitors, $\alpha 1$ adrenoreceptors blockers and phytotherapies, like saw palmetto (SP) lipid extracts. D-004, a lipid extract of Roystonea regia fruits, reduced experimental prostate hyperplasia in rodents and the International Prostate Symptoms Score (IPSS) as effectively as SP in a pilot trial in men with BPH.
\end{abstract}

Objective: To compare the effects of D-004 and SP in the treatment of men with symptomatic BPH.

Methods: Sixty-one patients with moderate BPH were double-blindly randomised to D-004 or SP $(320 \mathrm{mg} / d a y$ both) capsules for 4 months. Decrease on IPSS was the primary efficacy variable. Effects on prostate size, residual volume post-voiding and serum prostate specific antigen (PSA) were secondary outcomes.

Results: After 2 months on therapy, D-004 and SP decreased ( $p<0.05)$ mean IPSS values by 2.1 units versus baseline (15.8\% and $16.0 \%$ decreases, respectively). This effect increased at study completion, when IPSS decreased by 4.8 (36.0\%) with D-004 and by 4.5 units (34.1\%) with SP ( $p<0.0001$ in both cases), without differences between groups. Both treatments did not modify prostate size, residual volume post-voiding. Treatment with $S P$, not with D-004, increased $(p<0.05)$ serum PSA. Two SP-treated patients withdrew from the study, one due to an adverse experience (hemorrhagic stroke). One D-004- and 2 SP-treated patients experienced adverse experiences.

Conclusions: D-004 (320 mg/day) for 4 months was as effective as SP (320 mg/day) for decreasing LUTS in men with moderate BPH, and well tolerated. Further studies should confirm these results.

KEY WORDS: benign prostate hyperplasia, D-004, IPSS, PSA, Roystonea regia, saw palmetto

\section{INTRODUCTION}

Benign prostatic hyperplasia (BPH), a common condition among older men, is a progressive disease that frequently produces undesirable lower urinary tract symptoms (LUTS) like weak urinary stream, frequency and urgency [1]. Pharmacological treatments for BPH includes 5 $\alpha$-reductase inhibitors, $\alpha 1$-adrenoreceptors (ADR) blockers and the combination therapy with drug of both therapeutic classes, which is significantly more effective for ameliorating the symptoms and prevent BPH progression than either agent alone [27].Complementary medicines have been widely used for decades to treat BPH/LUTS [8], mainly the lipid extracts of saw palmetto (SP) (Serenoa repens) fruit, which contain a mixture of fatty acids, mainly oleic, lauric and myristic acids [8-10]. Despite some negative results [11,12], the use of SP for treating BPH is still documented $[2,10,13-17]$. The efficacy of SP in BPH is associated to a multifactorial mechanism including the inhibition of prostate $5 \alpha$-reductase, the antagonism of $\alpha 1$-ADR, anti-inflammatory and antioxidant effects, as well [18-23]. Also, the safety and tolerability of SP has been supported [24].

D-004, a lipid extract of the Roystonea regia (Arecaceae fam) fruits also contains a mixture of fatty acids, oleic, lauric, palmitic and myristic being the most abundant. Experimental studies demonstrated that D-004 decreased testosterone (T)-induced prostate hyperplasia in rodents [25-28], inhibited rat prostate $5 \alpha$ reductase in vitro [29], antagonized $\alpha 1$-ADR-mediated responses [30,31], and produced antioxidant effects in the prostate tissue of normal and testosterone-treated rats [32,33]. Clinical studies have demonstrated that D-004 
produced beneficial antioxidant effects on plasma oxidative variables in healthy and BPH men [34,35], and this last pilot trial revealed that D-004 reduced significantly LUTS assessed through the international prostate symptom score (IPSS) [36], and similarly to SP. In light of this background, this study was undertaken to confirm whether the effects of D-004 (320 mg/day) on BPH symptoms, measured by the IPSS [37], were comparable to those of SP $(320 \mathrm{mg} / \mathrm{day})$.

\subsection{Study Design}

\section{MATERIALS AND METHODS}

This randomized, double-blinded, comparative study was conducted in accordance with the Declaration of Helsinki. The study protocol and procedures was approved by the Institutional Committee for Clinical Research of the Salvador Allende Hospital. Also, the Cuban State Drug Control Centre (Havana City, Cuba). Approved the study protocol and staff of such centre independently audited the study conduction. All participants provided written informed consent at enrolment. After obtaining their consent, men aged $\geq 50$ years and previously diagnosed with BPH were recruited from the Urology Department of the Hospital and from the surrounding suburbs by contacts to patients and letters to their Family Doctors. At enrolment they underwent clinical history, IPSS questionnaires and physical examination for screening their eligibility for randomization (visit 1). Only BPH patients with IPSS values of 7 or more, but below 19, and without exclusion criteria were randomized to D-004 or SP $(320 \mathrm{mg})$ soft gel capsules once daily for 4 months. Randomized patients were advised to continue on their usual dietary habits. Further visits were done after complete 1, 2, 3, and 4 months on therapy (visits 3 to 6). Subjects underwent physical examination and IPSS interview at each visit. Treatment compliance and adverse experiences (AE) were controlled from visits 3 to 6 . Laboratory tests and ultrasound evaluation were conducted at baseline and at study completion.

\subsection{Study subjects}

BPH patients aged $\geq 50$ years were enrolled in the trial. To be eligible for randomization, enrolled men should have moderate BPH/LUTS (IPSS $\geq 7,<19)[37,38]$ and should not show any of the exclusion criteria summarised below. Digital rectal prostate examination confirmed participants' eligibility for the study. Patients with any major prostate disease except BPH or those who had urogenital surgery, urinary retention, neurogenic bladder, had a prostate specific antigen (PSA) level $>5.0 \mathrm{ng} / \mathrm{dL}$; and/or a creatinine level $>177 \mu \mathrm{mol} / \mathrm{L}$ were excluded from the study. Likewise, patients were ineligible if they had arterial pressure values $>180 / 110 \mathrm{mmHg}$, psychiatry problems that limited proper answers to the IPSS questionnaire, diagnosed neoplasias, serious events (acute coronary syndromes, stroke, transient ischemic attacks, major surgery, among others) during the prior 6 months and/or receiving BPH/LUTS-related therapy (5 $\alpha$-reductase inhibitors, $\alpha 1$-adrenoreceptor blockers, or phytotherapy).

Causes of premature discontinuations were to experience any $\mathrm{AE}$ justifying such a decision, unwillingness to continue on the trial and major violations (failure in taking study treatments for $\geq 5$ days and/or to consume supplements or medicines with known effects on BPH/LUTS).

\subsection{Treatments}

The extracts were manufactured in one batch each to ensure same consistencies over the trial. The capsules of D-004 and SP (Rainbow \& Nature, Ltd, Sydney, Australia) had the following free fatty acid composition, assessed with a validated gas chromatography method: D-004 capsules: caprylic $0.4 \%$, capric $0.6 \%$, lauric $21.9 \%$, myristic $10.8 \%$, palmitic $10.5 \%$, palmitoleic $0.3 \%$, stearic $2.3 \%$, oleic $43.3 \%$, with a purity (total of fatty acids) of $90.1 \%$. SP capsules: caprylic $1.5 \%$, capric $2.5 \%$, lauric $29.9 \%$, myristic $11.8 \%$, palmitic $8.2 \%$, palmitoleic $0.2 \%$, stearic $1.9 \%$, oleic $33.3 \%$, with a purity of $89.4 \%$. Also, D-004 and SP extracts had $0.02 \%$ and $0.33 \%$ of sterols, respectively.

The dose (320 mg/day) was selected because it is that most documented for SP [10-14]. Also, D-004 administered at this dose for short-term ( 8 weeks) was able to produce antioxidant effects in healthy and BPH men [34-36], and to lower IPSS values of these last ones in a previous pilot trial [36]. D-004 and SP capsules, identical on appearance and packaged in identical codified containers, were given to the subjects according to their serial progressive inclusion. Randomisation was computer-generated using balanced blocks and allocation ratio 1:1, without stratification. The randomization list was created by personnel who were not associated with the trial. The study treatments were dispensed in numbered bottles (provided by the manufacturer), according to the randomization sequence. Study participants and the personnel who administered interventions, assessed outcomes, or performed data analysis ignored the treatment allocation and the randomized sequence list. 
Patients were advised to take the study medication once a day with the breakfast for 4 months, and to bring all unused capsules to each study visit. Treatment compliance was assessed by counting the remainder capsules and interviewing the men. Compliance was considered as good if the subjects taken at least $80 \%$ of the capsules scheduled from the previous visit, and very good if consumption was over $90 \%$.

Consumption of medications and/or supplements with known effects on BPH/LUTS or/and urination were not allowed during the study. Subjects who were taking some of them were eligible for randomisation only if they discontinued consumption for at least 6 months prior to the trial.

\subsection{Efficacy variables}

A significant reduction of IPSS was the primary efficacy variable. Since a decrease of 4 IPSS points has been suggested as a clinically meaningful symptom reduction, the treatments were considered effective if they reduced significantly mean IPSS values by at least 4 points as compared to baseline [2], and comparable if the final IPSS values and IPSS net changes in both groups were statistically similar. IPSS was assessed using a standard questionnaire form with seven questions, each measured on a scale to which patients respond from 0 (the best) to 5 (the worst) [37,38]. Secondary efficacy outcomes included prostate size, measured by transrectal ultrasonography; residual volume after voiding, measured by diagnostic ultrasound; and changes in PSA levels. As for the primary outcome, treatment efficacy was judged in accordance to the significant reduction of values versus baseline, the treatments being considered similarly effective if no significant differences between groups were found.

\subsection{Safety and tolerability}

Data from physical examination, laboratory safety indicators and adverse experiences (AE) were analysed. Safety indicators included physical (body weight, pulse rate and blood arterial pressure), haematological (haemoglobin, hematocrit, platelets, red cells and white cell counts) and blood biochemistry safety indicators (alanine amino transferase -ALT-, aspartate amino transferase -AST-, glucose, creatinine, total cholesterol (TC) and triglycerides (TG).

All undesirable events occurred to a subject during the trial, disregarding the cause, should be considered as $\mathrm{AE}$, whenever they newly appeared during the trial. In accordance with their intensity, AE were classified as mild, moderate or serious. Mild AE should not require suspension of study capsules and/or specific treatment of the $\mathrm{AE}$, moderate $\mathrm{AE}$ should require stopping therapy and/or specific treatment of the $\mathrm{AE}$, serious AE should lead to hospitalisation and/or deaths.

\subsection{Laboratory variables}

Blood venous samples were drawn after an overnight fast of 8-12 hours. Haematological indicators were automatically determined in the Haematological Complex equipment. Blood biochemistry indicators were assessed using reagent kits (Roche, Switzerland) in the Hitachi 912 autoanalyser (Tokyo, Japan). PSA levels were determined by immunoenzymatic ELISA (UMELISA PSA kit, Immunoassay Centre, La Habana, Cuba).

\subsection{Statistical analysis}

A sample size of 30 subjects/treatment group was expected to provide $80 \%$ power to detect a $20.0 \%$ between-group difference in the mean change from baseline in IPSS. Data analyses were performed in accordance to intention to treat (ITT), including all randomized subjects, regardless of study treatment compliance. Assuming a $15 \%$ of premature withdrawals, approximately 66 patients should be enrolled, so that a minimum of 70 subjects should be enrolled. No interim analyses were planned or done.

Comparisons of continuous variables were performed using the $t$ tests for paired (within group comparisons) and independent samples (between group comparisons). Categorical variables were compared with the two tailed Fisher's Exact Test and confirmed by the chi-square test. A value of $\alpha=0.05$ was assumed for statistical significance. Comparisons were done with the Statistics software for Windows (USA).

\subsection{Baseline characteristics of study subjects}

\section{RESULTS}

Of 80 enrolled patients 61 were randomised to D-004 $(n=31)$ or SP $(n=30)$, while 19 were not eligible because of IPSS values below (10 men) and above (1) the inclusion criteria, PSA values $>5 \mathrm{ng} / \mathrm{mL}$ (2) and further decline to attend to the inclusion (randomization) visit (6 men). Fifty-nine (59) of 61 randomized subjects $(96.7 \%)$ completed the study. The two withdrawals, both from the SP group, were due were due to unwillingness to follow (1) and a serious AE (fatal hemorrhagic stroke), respectively.

Both groups were well balanced at baseline (Table 1). Main co-morbid diseases were hypertension 
(50.8\%), overweight (42.6\%), dyslipidemia (41.0\%), diabetes (13.1\%) and obesity (6.6\%). Undesirable lifestyle habits like smoking and alcoholism accounted for $21.3 \%$ and $4.9 \%$, respectively. The frequency of concomitant medications, well matched in the two groups, was high $(70.5 \%)$. The most frequent concomitant therapies were antihypertensive drugs (diuretics, angiotensin converting enzyme inhibitors -ACEI-, $\beta$-blockers, calcium antagonists), followed by antiplatelet, oral hypoglycemic and lipid-lowering drugs. With the exception of the two withdrawals, all randomized patients consumed $100 \%$ of the scheduled dose. Then, treatment compliance was very good and similar in both groups.

\subsection{Effects on the primary outcome}

After 2 months on therapy, both D-004 and SP decreased significantly ( $\mathrm{p}<0.001)$ mean IPSS values by 2.1 points as compared to baseline; $(15.8 \%$ and $16.0 \%$ decreases, respectively). This effect increased at study completion, when IPSS decreased by 4.8 (36.0\%) with D-004 and by 4.5 units $(34.1 \%)$ with SP (p<0.0001 in both cases), without significant difference between both groups. At study completion, the proportions of D-004 and SP-treated patients whose IPSS values were reduced by at least 4 units were statistically similar (19 in each group). The same was true for those who achieved symptom relief (IPSS <7): 14/31 (45.2\%) with D-004, 11/30 $(36.7 \%)$ with SP.

\subsection{Effects on secondary outcomes}

Both treatments failed to change the prostate volume and the residual volume after voiding as compared to baseline. Treatment with SP, not with D-004, increased $(\mathrm{p}<0.05)$ serum PSA values $($ Table 3$)$. Comparisons of the secondary outcomes also revealed that treatments were comparable

\subsection{Safety and tolerability}

Both treatments were well tolerated. No significant impairment of physical or blood safety indicators were found, all individual values remaining within normal range (values not shown for simplicity).

Only two SP-treated patients withdrew from the study, one due to a serious AE (hemorrhagic stroke). Three of 61 patients (4.9\%) experienced AE during the study. One patient of the D-004 group had flu-related pneumonia, and 2 SP-treated cases had hemorrhagic stroke (1 patient) and flatulence plus pruritus (1 patient).

\section{DISCUSSION}

In this 4 months randomized trial we found that D-004 was similarly effective as SP for improving urinary symptoms in patients with mild to moderate BPH. Also, both treatments reduced prostate size, but unchanged residual volume post-voiding as compared to baseline, while serum PSA values unchanged with D-004, meanwhile increased with SP. Both groups had similar baseline characteristics, being homogeneous for comparisons. Study patients had moderate BPH (IPSS $\geq 7,<19$ ), and concomitant morbidities (hypertension, overweight, dyslipidemia, diabetes, obesity) characteristics of this population [39,40]. Concomitant medications were consistent with the personal history of the patients.

Keeping in mind that SP was the reference comparator, it is relevant to remark that the product used in this trial complied with the recommendation that SP extracts should contain 70 to $95 \%$ of fatty acids and 0.2 to $0.5 \%$ sterols [10]. The extract here used had $88.4 \%$ fatty acids and $0.33 \%$ sterols. The mean reductions of IPSS (4.8 points with D-004, 4.5 with SP) were greater than 4 points, so that the changes on BPH symptoms can be considered as clinically meaningful. The reduction of IPSS here seen was similar to that found in a 6 month study where SP decreased significantly the mean IPSS from 16.7 to 12.3 as compared to baseline, the difference being significant versus placebo too [41], but greater than that found in one year multinational study (3.2 decrease) [2]. On its side, the effect of D-004 (320 mg/day) was quite similar to that found when it was given for 2 months to patients with moderate BPH (net decrease 4.45, 33.9\% reduction) [36]. Nevertheless, the efficacy at month 2 on treatment was frankly inferior to that reported for the same treatment period [36], despite the baseline characteristics -including IPSS values- of study patients, D-004 composition and treatment compliance were similar in both studies. Then, we do not find a conclusive explanation for such a difference.

Since the effects of both treatments on IPSS values at study completion (4 months) were similar we may conclude that D-004 was as effective as SP for ameliorating LUTS on these patients. These results suggest, however, that the $20 \%$ difference between treatments assumed for the sample size calculation was overestimated, so that the present results should be confirmed in studies conducted in larger sample sizes.

Various action mechanisms are suggested for the efficacy of D-004 for treating BPH/LUTS, mainly including its inhibition on 5 $\alpha$-reductase [29] and its antagonism on $\alpha 1$-ADR-mediated responses [30,31], as well as its inhibition of 5-lipoxygenase pathway [42] and its antioxidant effects [32-35].

The effects of both treatments on prostate size and the residual post-voiding volume were not significant, in agreement with most published for SP that found no effect or just small significant reductions 
[9,10,43-45]. Nevertheless, it should be noted that the great dispersion of baseline and after treatment values of these variables could limit the possibility of find any significant change in our study. The high variability of these data, however, matches with the results found in real clinical practice. One limitation of the present study was that effects on maximal urinary flow, a relevant urological outcome, were not assessed, which prevented the demonstration of effects on another objective variable. Unexpectedly, serum PSA increased with SP, while remained unchanged with D-004. The effects of SP on PSA have been some controversial, most studies have documented that SP does not modify PSA levels $[2,9,10,45]$, while some trials have found mild significant reductions [44]. To our knowledge, this is the first study reporting an increase of serum PSA after SP therapy.

The treatments were safe and well tolerated, consistent with previous data on SP [11-24] and D-004. The hemorrhagic stroke that occurred in one SP-treated patient was considered as not related to the treatment but to the prior history of the patient (coronary heart disease, hypertension). The present results merit continue further research of the effects of D-004 on patients with BPH/LUTS, including studies with larger sample sizes, longer treatment and searching effects on the urinary flow.

\section{CONCLUSIONS}

In this study, D-004 (320 mg/day) given for 4 months was as effective as SP (320 mg/day) for decreasing LUTS in men with moderate BPH. Both treatments were well tolerated. Further studies should confirm the efficacy of D-004 for BPH.

\section{REFERENCES}

[1]. C.G. Roehrborn, Male lower urinary tract symptoms (LUTS) and benign prostatic hyperplasia (BPH), Med Clin North Am, 95, 2011,87-100.

[2]. A. Hutchison, R. Farmer, K. Verhamme, R. Berges, R. Vela. The efficacy of drugs for the treatment of LUTS/BPH. A study in 6 European countries, European Urology, 51, 2007, 207-216.

[3]. X.S. Liu, C. Folia, L.G. Gomella, Pharmacology for common urologic diseases: 2011 review for the primary care physician, Can J Urol, 18 Suppl, 2011, 24-38.

[4]. R.F. Donnell, Benign prostate hyperplasia: a review of the year's progress from bench to clinic, Curr Opin Urol, 21, 2011, 22-26.

[5]. F. Azzouni, J. Mohler, Role of 5 $\alpha$-reductase inhibitors in benign prostatic diseases, Prostate Cancer Prostatic Dis, 15, 2012, 222230.

[6]. J. Yuan, Y. Liu, Z. Yang, X. Qin, K. Yang, C. Mao, The efficacy and safety of alpha-1 blockers for benign prostatic hyperplasia: an overview of 15 systematic reviews, Curr Med Res Opin, 29, 2013, 279-87.

[7]. S.A. Cohen, J.K. Parsons, Combination pharmacological therapies for the management of benign prostatic hyperplasia, Drugs Aging, 29, 2012, 275- 284.

[8]. H. Azimi, A.A. Khakshur, I. Aghdasi, M. Fallah-Tafti, M. Abdollahi, A review of animal and human studies for management of benign prostatic hyperplasia with natural products: perspective of new pharmacological agents, Inflamm Allergy Drug Targets, $11,2012,207-221$.

[9]. P.M. Barnes, E. Powell-Griner, K. McFann, R.L. Nahin, Complementary and alternative medicine use among adults: United States, 2002. Adv Data, 43, 2004, 1-19.

[10]. Pharmacopeial Convention, Saw palmetto extract In: Expert Comitee. United Status pharmacopeial forum: (DSB) dietary supplement: botanicals. Rockville, Md. Pharmacopeial convention 28, 2005, 425-426.

[11]. S. Bent, C. Kane, K. Shinohara, Saw Palmetto for Benign Prostatic Hyperplasia, NEJM,354, 2006, 557-566.

[12]. M.J. Barry, S. Meleth, J.Y. Lee, K.J. Kreder, A.L. Avins, J.C. Nickel, C.G. Roehrborn, E.D. Crawford, H.E. Foster Jr, S.A. Kaplan, A. McCullough, G.L. Andriole, M.J. Naslund, O.D. Williams, J.W. Kusek, C.M. Meyers, J.M. Betz, A. Cantor, K.T. McVary, Complementary and Alternative Medicine for Urological Symptoms (CAMUS) Study Group, JAMA, 306, 2011, 13441351.

[13]. J. Tacklind, R. Macdonald, I. Rutks, J.U. Stanke, T.J. Wilt, Serenoa repens for benign prostatic hyperplasia. Cochrane Database Syst Rev. 2012 Dec 12; 12:CD001423. doi: 10.1002/14651858.CD001423.pub3.

[14]. F. Mantovani, Serenoa repens in benign prostatic hypertrophy: analysis of 2 Italian studies, Minerva Urol Nefrol, 62, 2010, 33540 .

[15]. I. Sinescu, P. Geavlete, R. Multescu, C. Gangu, F. Miclea, I. Coman, I. Ioiart, V. Ambert, T. Constantin, B. Petrut, B. Feciche, Long-term efficacy of Serenoa repens treatment in patients with mild and moderate symptomatic benign prostatic hyperplasia, Urol Int, 86, 2011, 284-289.

[16]. S. Spatafora, A. Casarico, A. Fandella, C. Galetti, R. Hurle, E. Mazzini, C. Niro, M. Perachino, R. Sanseverino, G.L. Pappagallo, RO.it BPH Guidelines Committee. Evidence-based guidelines for the treatment of lower urinary tract symptoms related to uncomplicated benign prostatic hyperplasia in Italy: updated summary from AURO.it. Ther Adv Urol, 4, 2012, $279-301$.

[17]. A. Bertaccini, M. Giampaoli, R. Cividini, G.L. Gattoni, R. Sanseverino, T. Realfonso, G. Napodano, A. Fandella, E. Guidoni, D Prezioso, R. Galasso, C. Cicalese, V. Scattoni, A. Armenio, G. Conti, M. Corinti, R. Spasciani, G. Liguori, N. Lampropoulou, G. Martorana, Arch Ital Urol Androl, 84, 2012, 117-22.

[18]. J.P. Raynaud, H. Cousse, P.M. Martin, Inhibition of type 1 and 25 alpha reductase activity by free fatty acids, active ingredients of Permixon, J Steroid Biochem, 82, 2000, 233-239.

[19]. F.K. Habib, M. Ross, C.K. Ho, Serenoa repens (Permixon) inhibits the 5 alpha-reductase activity of human prostate cancer cell lines without interfering with PSA expression. Int J Cancer, 114, 2005, 190-194.

[20]. M. Abe, Y. Ito, L. Oyunzul, T. Oki- Fujino, S. Yamada, Pharmacologicall Relevant receptor Binding Characteristcs and 5 $\alpha-$ reductase inhibitory activity of free fatty acids contained in Saw Palmetto Extract, Biol Pharm Bull, 32, 2009 , 646-650.

[21]. P. Pais, Potency of a novel saw palmetto ethanol extract, SPET-085, for inhibition of 5alpha-reductase II, Adv Ther, 27, 2010, 555-563.

[22]. M. Goepel, L. Dihn, M. Michel, Do Saw palmetto extracts block human alfa 1 adrenoceptors subtypes in vivo? Prostate, 46, 2006, 226-232.

[23]. L.I. Belostottskaia, I.V. Nikitchenko, O.N. Gomon, L.A. Chaika, V.V. Bondar, V.N. Dziuba, Effect of biologically active 
substances of animal and plant origin on prooxidant-antioxidant balance in rats with experimental prostatic hyperplasia, Eksp Klin Farmakol, 69, 2006, 66-68.

[24]. A.L. Avins, S. Bent, S. Staccone, E. Badua, A. Padula, H. Goldberg, J. Neuhaus, E. Hudes, K. Shinohara, C. Kane, A detailed safety assessment of a saw palmetto extract, Complement Ther Med, 16, 2008, 147-154.

[25]. M.L. Arruzazabala, D. Carbajal, R. Mas, V. Molina, V. González. E. Rodríguez, Preventive effects of D-004, a lipid extract from Cuban royal palm (Rostoynea regia) fruits, on prostate hyperplasia induced with testosterone on intact and castrated rodents, Drugs Exptl Clin Res, 30, 2004, 227 - 234.

[26]. D. Carbajal, M.L. Arruzazabala, R. Mas, V. Molina, E. Rodriguez, V. Gonzalez, Effect of D-004, a lipid extract from Cuban royal palm fruit, on inhibiting prostate hyperplasia induced with testosterone and dihydrotestosterone in rats, Curr Ther Res Clin \& Exptl, 65, 2004, $505-514$.

[27]. M. Noa, M.L. Arruzazabala, D. Carbajal, R. Mas, V. Molina, Effect of D-004, a lipid extract from Cuban royal palm fruit, on histological changes of prostate hyperplasia induced with testosterone in rats, Int J Tiss React, 27, 2005,193 - 198.

[28]. D. Carbajal, V. Molina, R. Mas, M.L. Arruzazabala, Therapeutic effect of D-004 a lipid extract from Roystonea regia fruits on prostate hyperplasia induced in rats, Drugs Exp Clin Res, 31, 2005, 193-197.

[29]. Y. Pérez, R. Menéndez, R. Mas, R. González R, In vitro effect of D-004, a lipid extract from the Cuban Royal palm (Roystonea regia) fruits, on the activity of prostate steroid 5-alpha reductase, Curr Ther Res Clin \& Exptl, 67, 2006, 396 - 405.

[30]. M.L. Arruzazabala, R. Mas, D. Carbajal, V. Molina, Effect of D-004, a lipid extract from the Cuban royal palm fruit, on in vitro and in vivo effects mediated by alpha-adrenoceptors in rats, Drugs $R \& D, 6,2005,281-289$.

[31]. M.L. Arruzazabala, R. Mas, V. Molina, M. Noa M, D. Carbajal, Effect of D-004, a lipid extract from the fruits of Cuban royal palm, on the atypical prostate hyperplasia induced with phenylephrine in rats, Drug $R \& D, 7,2006,233-234$.

[32]. R. Menéndez, R. Mas, Y. Pérez, R.M. González, In vitro effect of D-004, a lipid extract of the ground fruits of the Cuban royal palm (Roystonea regia), on rat microsomal lipid peroxidation, Phytother Res, 21, 2007, 89-95

[33]. Y. Pérez, V. Molina, R. Mas, R. Menéndez R, et al, Ex vivo antioxidant effects of D-004, a lipid extract from Roystonea regia fruits, on rat prostate tissue, Asian J Androl, 10, 2008, 659- 666.

[34]. E. Lopez, V. Molina, J. Illnait, A. Oyarzábal, R. Mas R, et al, Antioxidant effects of D-004, a lipid extract from the Roystonea regia fruits, on the plasma of healthy men, Asian J Androl, 11, 2009, 385-392.

[35]. I. Rodríguez, V. Molina, R. Mas, J. Illnait, A. Oyarzábal, S. Mendoza, L. Fernández, J. Fernández, R. Gámez, M. Mesa, S. Jiménez, L. Borrero, Y. Cruz, Y. Perez. Comparison of the antioxidant effects of lipid extracts of Roystonea regia (D-004) and saw palmetto on blood oxidative variables of healthy men. Lat Am J Pharm, 29, 2010, 1185-1192.

[36]. R. Guzmán, J. Illnait, R. Mas, V. Molina, Y. Perez, L. Fernández, S. Mendoza, A. Oyarzábal, J. Fernández, R. Gámez, M. Mesa, L. Borrero, S. Jiménez, D. Ruiz, P. Reyes P, Comparative effects of Roystonea regia (D-004) and Saw palmetto lipid extracts on blood oxidative variables in men with benign prostate hyperplasia (BPH), IOSR PHR 2013 (in press).

[37]. T. Fujimura, H. Kume, H. Nishimatsu, T. Sugihara, A. Nomiya, Y. Tsurumaki, H. Miyazaki, M. Suzuki, H. Fukuhara, Y. Enomoto, Y. Homma. Assessment of lower urinary tract symptoms in men by international prostate symptom score and core lower urinary tract symptom score. BJU Int, 109, 2012, 1512-1516.

[38]. X. Badia, M. García, R. Dalre, Ten language translation and harmonization of the International Prostate Symptom Score: Developing a methodology for multinational clinical trials, Eur Urol, 31, 1997, 129-131.

[39]. I.H. Chen, Y.S. Tsai, Y.C. Tong, Correlations among cardiovascular risk factors, prostate blood flow, and prostate volume in patients with clinical benign prostatic hyperplasia, Urology, 79, 2012, 409-414.

[40]. J.K. Parsons, Lifestyle factors, benign prostatic hyperplasia, and lower urinary tract symptoms, Curr Opin Urol, 21, $2011,1-4$.

[41]. G.S. Gerber, D. Kuznetsov, B.C. Johnson, J.D. Burstein, Randomized, double-blind, placebo-controlled trial of saw palmetto in men with lower urinary tract symptoms, Urology, 58, 2001, 960-964.

[42]. R. Menéndez, D. Carbajal, R. Mas, Y. Pérez, V. Molina, M.L. Arruzazabala, R. González, Efectos del D-004, extracto lipídico de los frutos de la palma real (Roystonea regia) sobre el granuloma inducido por algodón en ratas y sobre la lipooxigenasa presente en leucocitos polimorfonucleares (PMNs), Acta Farm Bonaerense, 25, 2006, 213-218

[43]. L.S. Marks, A.W. Partin, J.I. Epstein, V.E. Tyler, I. Simon, M.L. Macairan, T.L. Chan, F.J. Dorey, J.B. Garris, R.W. Veltri, P.B. Santos, K.A. Stonebrook, J.B. deKernion, Effects of a saw palmetto herbal blend in men with symptomatic benign prostatic hiperplasia, J Urol, 163, 2000, 1451-1456.

[44]. H. Hong, C. Kim, S. Maeng, Effects of pumpkin seed oil and saw palmetto oil in Korean men with symptomatic benign prostatic hyperplasia, Nutr Res Pract, 3, 2009, 323-327.

[45]. F. Debruyne, G. Koch, P. Boyle, F.C. Da Silva, J.G. Gillenwater, F.C Hamdy, P. Perrin, P. Teillac, R. Vela-Navarrete, J.P. Raynaud, Comparison of a phytotherapeutic agent (Permixon) with an alpha-blocker (Tamsulosin) in the treatment of benign prostatic hyperplasia: a 1-year randomized international study, Eur Urol, 41, 2002, 497-506. 
Table 1. Main baseline characteristics of study subjects

\begin{tabular}{|c|c|c|c|}
\hline Characteristics & D-004 (320 mg/day) $(\mathrm{n}=31)$ & SP (320 mg/day) $(n=30)$ & Total $(n=61)$ \\
\hline \multicolumn{4}{|c|}{$(\mathrm{X} \pm \mathrm{SD})$} \\
\hline Age (years) & $65 \pm 6$ & $63 \pm 8$ & $64 \pm 7$ \\
\hline BMI $\left(\mathrm{kg} / \mathrm{m}^{2}\right)$ & $25.5 \pm 3.8$ & $25.2 \pm 2.6$ & $25.4 \pm 3.3$ \\
\hline IPPS & $13.3 \pm 3.7$ & $13.3 \pm 3.4$ & $13.3 \pm 3.53$ \\
\hline \multicolumn{4}{|c|}{ Personal history (n) } \\
\hline Hypertension & 16 & 15 & 31 \\
\hline Overweight $\left(\mathrm{kg} / \mathrm{m}^{2} \geq 25,<30\right)$ & 11 & 15 & 26 \\
\hline Dyslipidemia & 13 & 12 & 25 \\
\hline Smoking & 8 & 5 & 13 \\
\hline Diabetes & 5 & 3 & 8 \\
\hline Obesity $\left(\mathrm{kg} / \mathrm{m}^{2} \geq 30\right)$ & 3 & 1 & 4 \\
\hline Alcoholism & 1 & 2 & 3 \\
\hline Coronary heart disease & 2 & 1 & 3 \\
\hline \multicolumn{4}{|c|}{ Concomitant therapy $(n)^{a}$} \\
\hline Any concomitant drug & 21 & 22 & 43 \\
\hline Diuretics & 9 & 6 & 15 \\
\hline ACEI & 4 & 8 & 12 \\
\hline$\beta$-blockers & 4 & 6 & 10 \\
\hline Calcium antagonists & 5 & 3 & 8 \\
\hline Antiplatelet drugs & 4 & 3 & 7 \\
\hline Oral hypoglycemic drugs & 4 & 3 & 7 \\
\hline Lipid-lowering drugs & 3 & 3 & 6 \\
\hline
\end{tabular}

SP- saw palmetto; X- mean; n- numbers; BMI- body mass index; IPPS- International Prostate Symptom Score; ACEI- angiotensin converting enzyme inhibitors, ${ }^{a}$ Consumed by $\geq 6$ patients.

All comparisons were not significant.

Table 2. Effects of D-004 and SP on IPSS values in patients with BPH $\left(\mathrm{X}^{1} \pm \mathrm{MSE}\right)$

\begin{tabular}{|c|c|c|c|c|c|}
\hline Treatment & Baseline & $\mathbf{2}$ months & net change & $\mathbf{4}$ months & net change \\
\hline D004 & $13.00 \pm 0.67$ & $10.00 \pm 1.02^{*}$ & $\mathbf{3 . 0 0}$ & $7.00 \pm 0.95^{* *}$ & $\mathbf{6 . 0 0}$ \\
\hline $\mathbf{9 5 \%}$ CI & $(11.9-14.6)$ & $(9.1-13.2)$ & & $(6.5-10.4)$ & \\
\hline SP & $13.00 \pm 0.62$ & $9.50 \pm 1.17^{*}$ & $\mathbf{3 . 5 0}$ & $8.00 \pm 1.05^{* *}$ & $\mathbf{5 . 0 0}$ \\
\hline $\mathbf{9 5 \%}$ CI & $(12.0-14.6)$ & $(8.8-13.6)$ & & $(6.6-10.9)$ & \\
\hline
\end{tabular}

SP- saw palmetto; $\mathrm{X}^{1}$ - median, MSE- mean standard error, CI- confidence interval.

Net changes- after treatment minus baseline values

$* \mathrm{p}<0.05, * * \mathrm{p}<0.0001$, Comparison with baseline ( $\mathrm{t}$ test for paired samples)

Between group comparisons were not significant ( $t$ test for independent samples and ANOVA) 
Table 3. Effects of D-004 and SP on secondary outcomes in men with BPH $\left(\mathrm{X}^{1} \pm \mathrm{MSE}\right)$

\begin{tabular}{|c|c|c|}
\hline Treatment & Baseline & 4 months \\
\hline \multicolumn{3}{|c|}{ Prostate size $\left(\mathbf{c m}^{3}\right)$} \\
\hline D004 & $40.00 \pm 3.68$ & $37.00 \pm 3.01$ \\
\hline SP & $34.50 \pm 4.40$ & $31.50 \pm 3.57$ \\
\hline \multicolumn{3}{|c|}{ Residual post-voiding volume $\left(\mathbf{c m}^{3}\right)$} \\
\hline D004 & $58.00 \pm 15.48$ & $50.00 \pm 16.81$ \\
\hline SP & $60.50 \pm 14.82$ & $68.50 \pm 14.91$ \\
\hline \multicolumn{2}{|c|}{ PSA (ng/mL) } \\
\hline D004 & $1.91 \pm 0.36$ & $1.51 \pm 0.43$ \\
\hline SP & $1.53 \pm 0.34$ & $1.77 \pm 0.55^{*}$ \\
\hline
\end{tabular}

SP- saw palmetto; $\mathrm{X}^{\mathrm{I}}$ - median, MSE- mean standard error, PSA- prostate specific antigen.

$* \mathrm{p}<0.05 ; * * \mathrm{p}<0.0001$. Comparison with baseline ( $\mathrm{t}$ test for paired samples) 